\begin{tabular}{|c|c|c|}
\hline Beitr. Ent. & Keltern & ISSN 0005 - 805X \\
\hline $54(2004) 2$ & S. 333-342 & 20.12 .2004 \\
\hline
\end{tabular}

\title{
Eine neue Art der Gattung Beris LATreILle, 1802 aus Mitteleuropa
}

\section{(Diptera: Stratiomyidae)}

Mit 9 Figuren

Jens-Hermann Stuke

Zusammenfassung

Beris strobli auctt. ist ein Artenkomplex, der die Arten Beris bauseri spec. nov., Beris strobli Dušek \& Rozkošný, 1968 und Beris latifacies Nagatomi \& Tanaka, 1972 (status rev.) umfasst.

\section{Summary}

Beris strobli auctt. is a species complex involving the species Beris hauseri spec. nov., Beris strobli DUšEK \& RozkoŠNứ, 1968 and Beris latifacies NAGATOMI \& TANAKA, 1972 (status rev.).

\section{Stichwörter}

Diptera, Stratiomyidae, Beris, spec. nov., Europa, Amerika

key words

Diptera, Stratiomyidae, Beris, spec. nov., Europe, America

\section{Einleitung}

Im Rahmen der faunistischen Erfassung der Stratiomyiden Niedersachsens (STUKE 2003) wurden im Harz mehrere Tiere einer Beris-Art gefunden, die mit den gängigen Bestimmungsschlüsseln nicht bestimmt werden konnten: Mit der Arbeit von RozkošnÝ (1982) werden die Tiere als Beris strobli Dušek \& Rozkošń̛, 1968 determiniert, die Genitalien weichen aber deutlich von den Abbildungen bei Dušek \& RoZKošnÝ (1968) sowie Rozkošný (1982) ab. Die Merkmale, die Brugge (2002) nutzt um die holländischen Beris strobli abzugrenzen, sind für die niedersächsischen Tiere aufgrund der Beinfärbung unzutreffend. Bei der Untersuchung weiteren Materials von Beris strobli wurde erkannt, dass unter Beris strobli auctores drei Arten zusammengefasst wurden. Im folgenden wird dieses Problem gelöst, indem eine Art neu beschrieben wird und die Abgrenzung der Arten voneinander dargestellt wird. 


\section{Beris bauseri spec. nov.}

(Fig. 3+7)

= "Beris strobli nom. nov." p. P. - DušEK \& ROZKošNÝ (1968)

= "Beris strobli Dušek \& Rozkošný" - Wooduey (1981)

= "Beris strobli Dušek \& Rozkošný" p. P. - RozkošNÝ (1982)

= "Beris strobli Dusek \& Rozkosny, 1968" - BrugGe (2002)

Holotypus: $10^{*}$ : (1) "Asten / de Berken / 22.V.1961 / V. S. v. d. Goot"; (2) "BERIS / chalybata Först. / det. v. d. Goot"; (3) "Beris strobli / Dusek \& Rozk of / det. B. Brugge 1980"; (4) "Holotypus / Beris hauseri / spec. nov. o/ Stuke det. 2004" [rotes Etikett]. [Unterstreichung: Handschrift; "/": Zeilenumbruch]

Der Holotypus wird in der Sammlung des Zoologischen Museum der Universität Amsterdam [ZMAN] aufbewahrt.

Paratypen: Deutschland: $10^{*}$ (08.V.1993, Hessen, Messel bei Darmstadt, leg. et coll. Hauser); 10 (17.V.1994, Hessen, Messel bei Darmstadt, $180 \mathrm{~m}$ NN, SE der Grube, leg Geller-Grimm, coll. Hauser); 1 (23.V.1992, Bayern, Schöngeising, Alter Einfang, Kellerbach, leg. Schacht, coll. Stuke); $30^{\star} 0^{\star}$ (22.V.2002, Bayern, FFB, Schöngeising, Jägereinfang, $550 \mathrm{~m}$, leg. Schacht, coll. Zoologische Staatssammlung München [ZSMN]); Niederlande: 1 (23.V.1961, Asten deBerken, leg. van der Goot, coll. ZMAN); 1 \% (12. V.1981, Breda, leg. B. van Aartsen, coll. ZMAN); 1 (7.V.1992, Breda, Liesbos, FT 1816, leg. van der Goot, coll. ZMAN); $40^{\star}$ o $^{\circ}$ (22.V.1964, Bunde, Bunder Bos, leg. van der Goot, coll. ZMAN); $10^{*}$ (10.V.1961, Bunde, Bunder Bos, leg. J. A. W. Lucas, coll. ZMAN); $10^{*}$ (29.V.1975, deSteeg, leg. B. van Aartsen, coll. ZMAN); $10^{\star}$ (17.V.1983, Didam, leg. B. van Aartsen, coll. ZMAN); 1 o (04.VI.1974, Dudemirdum, leg. J. A. W. Lucas, coll. ZMAN); $1 \%$ (12.V.1968, Gilze Rijen, Hulten, leg. H. J. v. d. Krift, coll. ZMAN); 10 (22.V.1979, Nunspeet, leg. B. van Aartsen, coll. ZMAN); 10 (21.V. 1974, Putten, leg. B. van Aartsen, coll. ZMAN); $10^{\star}$ (30.V.1972, dito); 10 (26.V.1976, Seelingen, leg. B. v.an Aartsen, coll. ZMAN); 1 o (05.VI.1983, Ughelen, leg. B. v.an Aartsen, coll. ZMAN); 1 o (29.V.1983, Wilde Pl. tuin Lelystad, leg. J. de Rond, coll. ZMAN); Tschechien: $10^{\star}$ (24.-27.V.2001, Podyji $1,5 \mathrm{~km}$ NW of Hananice, damp forest, $300 \mathrm{~m}, 48^{\circ} 48^{\prime} 12^{\prime \prime} \mathrm{N}, 15^{\circ} 58^{\prime} 22^{\prime \prime} \mathrm{E}$, leg. Barták et Kubuk, coll. Rozkošný); Schweden: $10^{*}$ (23.VI.1999, Lule lappmark, Kvikkjokk, $315 \mathrm{~m}$, along Taraätno river, RN 7428-1581, leg et coll. J. M. \& W. van Steenis).

weiteres Material (Weibchen und Männchen ohne Genital): Deutschland: 1 \& (01.18.VI.2002, Sachsen, Weißeritzkreis, NSG "Hofehübel" Malaisefalle, $670 \mathrm{~m} \mathrm{NN}$, leg. et coll. Kehlmaier); Niederlande: 1 ㅇ (08.VI.1996, Kuinre, Kuinderbosamersf. c. 182535, leg. et coll. W. van Steenis); 1 (22.V.1961, Asten, de Berken, leg. van der Goot, coll. ZMAN); 1 q (10.VI.2001, Kraggenburg, Voorsterbos, 193-519, leg. et coll. W. van Steenis); 1 우 (14.V.2000, Den Dolder, Dennendal-noord, amersf. c. 145-462, leg. et coll. W. van Steenis); Slowakei: $1 \sigma^{*}$ (19.VI.1959, Vihoriat, Snirský kameň, leg. et coll. Rozkošný); 10 (30.V.1973, Velká Fatra, Lubochňa, leg. et coll. Rozkošný); Tschechien: 1 o (12.-16.VI.1962, Bítov přegrada, leg. et coll. Rozkošný).

\section{Beschreibung des Holotypus ( $\left.\sigma^{*}\right)$}

Länge 4,7 mm (gemessen von Fühlerbasis bis Abdomenspitze).

Kopf: Fühler schwarz; Innenseite der 8 Glieder des Flagellums mit metallisch glänzender Behaarung; übrige Behaarung schwarz bis bräunlich; Scapus und Pedicellus mit 
einzelnen schwarzen Borsten. Augen rotbraun; Facetten nach ventral kleiner werdend; Augenbehaarung hellbraun, nach anterodorsal länger werdend, die maximale Länge entspricht der Länge des Scapus. Dorsale Berührungslinie der Augen ctwa viermal so lang wie Ocellenhöcker. Ocellen orange. Ocellenhöcker schwarz behaart. Mittelfurche der Stirn erstreckt sich über etwa $1 / 3$ der Stirnlänge. Stirn und Gesicht glänzend schwarz; vollständig bedeckt mit nach ventral länger werdender schwarzer Behaarung, die maximal etwa die doppelte Scapus-Länge erreicht. Postcranium dorsal mit eng anliegender schwarzer Behaarung und heller Bestäubung, nach ventral mit abstehenden und länger werdenden schwarzen Haaren und unbestäubt. Rüssel gelbbraun.

Thorax: Thorax schwarz glänzend, nur Postalarcalli bräunlich. Integument bis auf den Hinterrand des Mesoscutums, das Scutellum und Subscutellum, Teile des Katepisternums und Teile des Anepisternum mit Punktgruben und daraus entspringenden schwarzen Haaren bedeckt. Scutellum mit vier Dornen; alle Dornen mit langen schwarzen Haaren. Flügel einheitlich braun getönt, vollständig mit Mikrotrichen bedeckt; Flügeladern außer dem Bereich der Media zwischen den Basalzellen R und $M$ braun; Flügellänge 4,7 mm (gemessen von Tegula bis Flügelspitze). Halteren gelbbraun. Beine bis auf die Spitzen der Femora (etwa $1 / 5$ gelb) und die Basis der Tibia (Tibia $1-2$ etwa $1 / 3$ gelb, Tibia 3 etwa $1 / 5$ gelb) braun; Femora und Hintertibie an der Unter- und Hinterseite mit Haaren, die die Länge des Tibiendurchmessers erreichen können. Empodien und Pulvillen schmutzig weiß. Klauen basal gelb, apikal schwarz.

Abdomen: Tergite mattschwarz; lateral mit langen schwarzen Haaren, dorsal mit kurzen hellgelben Haaren. Sternite glänzendschwarz; mit kurzen hellgelben Haaren. Genitalien eines Paratypus sind in Fig. 3 dargestellt. Diese entsprechen den Genitalien des Holotypus.

\section{Variabilität Männchen}

Die fünf männlichen Paratypen entsprechen der Beschreibung des Holotypus mit wenigen Ausnahmen: Die Länge der Stirnfurche kann bis zu $2 / 3$ der Stirnlänge erreichen. Es können zwei zusätzliche äußere Dornen am Scutellum vorkommen. Der Paratypus aus Schweden ist lateral am Abdomen hell behaart. Die Körperlänge liegt zwischen 4,7 und $6 \mathrm{~mm}$, die Flügellänge variiert zwischen 4,4 und 4,9 $\mathrm{mm}$.

\section{Beschreibung Weibchen}

Die Weibchen von Beris hauseri unterscheiden sich in folgenden Merkmalen von den Männchen:

Kopf: Augen mit gleichgroßen Facetten; Behaarung deutlich kürzer als Scapus und spärlicher als bei Männchen. Augen weit getrennt (Gesichtsbreite auf Höhe der Fühler : Gesamtbreite des Kopfes $=1: 2,6-1: 3,0)$. Kopf vollständig hellgelb behaart, nur oberhalb der Fühler ein kleiner Bereich unbehaart. Occiput mit hellbrauner Behaarung und dichterer hellgelber Bestäubung, ventral ohne abstehende Haare.

Thorax: Thorax grünmetallisch glänzend, die Behaarung ist ausschließlich goldfarben. Flügel hellbraun gefärbt; Flügeladern basal gelblich. Halteren hellgelb. Beine höchstens sehr schwach braun, überwiegen gelb. Basitarsi mindestens $\mathrm{zu}^{3 /}{ }_{4}$ gelb.

Abdomen Tergit 1 braun und bestäubt; Tergite 2-7 braun, bis auf den Vorderrand glänzend; lateral mit langen hellgelben Haaren. Sternite glänzend braun. Genitalfurca entsprechend Fig. 7. 

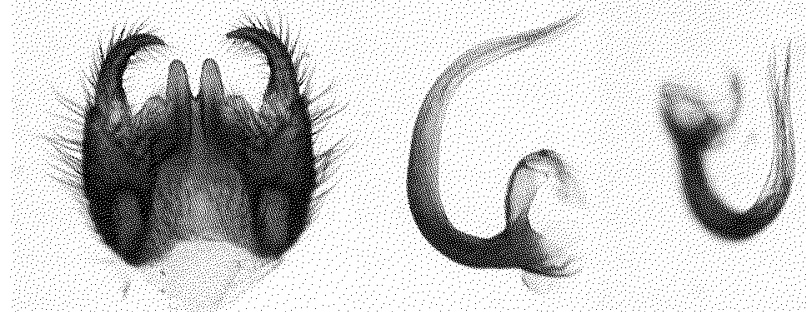

2
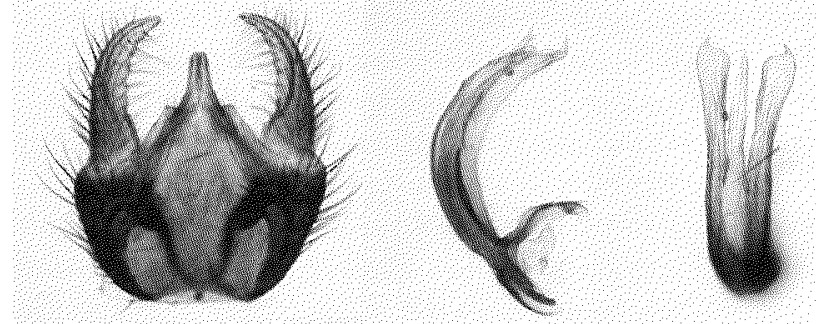

3
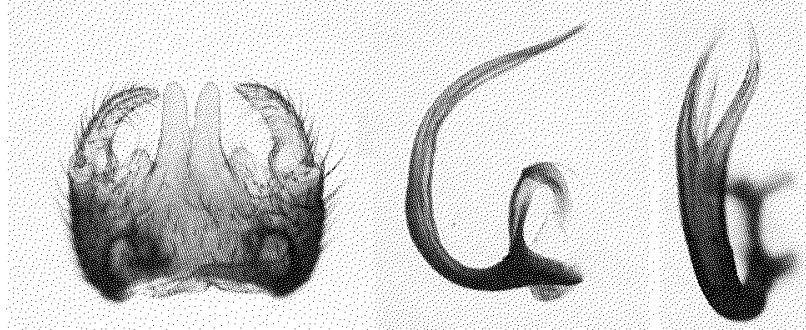

4
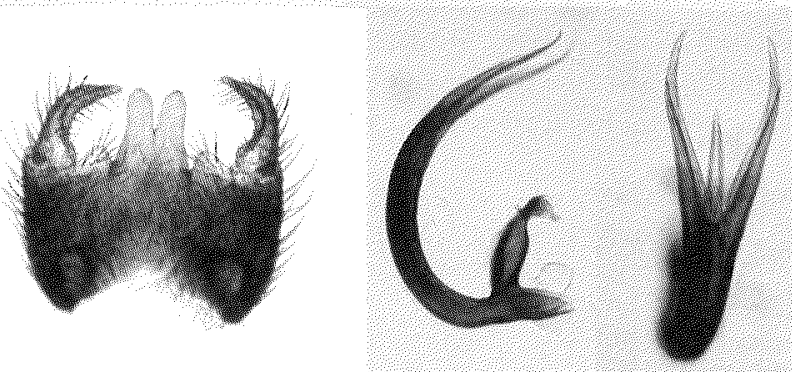

5
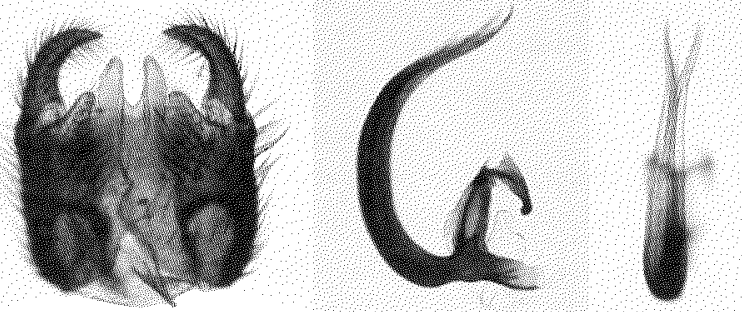


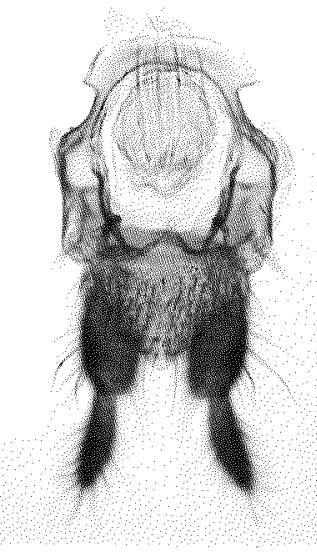

6

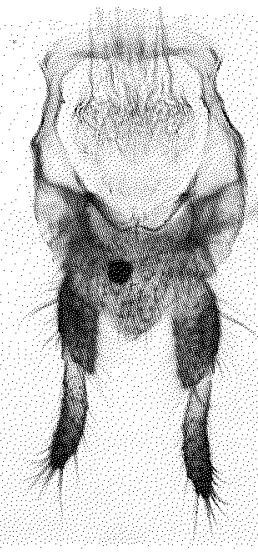

7

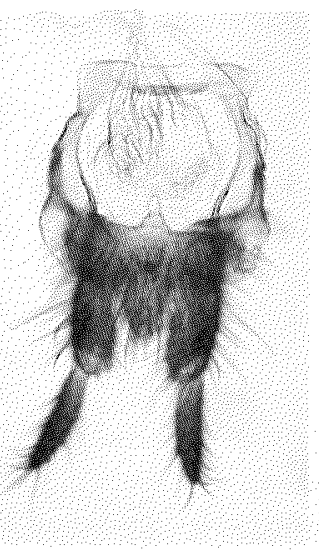

8

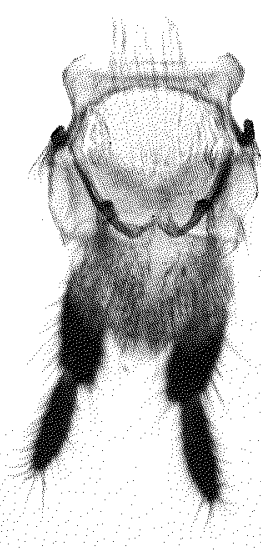

9

Fig. 6-9: Genitalfurka verschiedener Beris-Arten. - 6: Beris chalybata (FORSTER, 1771) [02.V.1999, D, Bremen, Neuenkirchen]; - 7: Beris hauseri spec. nov. [01.-18.VI.2002, D, Sachsen, NSG Hofehübel]; - 8: Beris latifacies Nagatomi \& Tanaka, 1972 [Paratypus, 04.VII.1963, Japan, Kai, Senjodake]; - 9: Beris strobli Dušer \& RozkošnÝ, 1968 [18.V.2002, D, Niedersachsen, Harz, Andreasbergertal].

\section{Derivatio nominis}

Die Art wird Martin Hauser (Urbana) gewidmet, einem der besten Kenner der Stratiomyiden. Ohne seine langjährige, freundschaftliche Hilfe und tatkräftige Unterstützung wäre diese Arbeit nicht möglich gewesen.

\section{Diagnose:}

Zur Gattung Beris LATrenlle, 1802 gehören weltweit 33 Arten, von denen in der Holarktis 26 Arten und in Europa 12 Arten vorkommen (Woodlex 2001). Von sämtlichen holarktischen Arten unterscheidet sich Beris hauseri nach den vorliegenden Beschreibungen (Kassebeer 1996, Nagatomi \& Tanaka 1972, Rozkošný 1982, Rozkošný \& Nartshuk 1980, Woodley 1981, Yang \& Nagatomi 1992) durch das mattschwarze Abdomen, das überwiegend schwarz behaarte Mesoscutum, die zumindest teilweise verdunkelten Beine und Basitarsi, das basal nicht verdickte Flagellum und die Form der männlichen Genitalien.

In Mitteleuropa bestehen Verwechselungsmöglichkeiten mit Beris chalybata (Forster, 1771), Beris geniculata Curtis, 1830 und Beris strobli Dušex \& Rozkošný, 1968. Mit dem Bestimmungsschlüssel können die Männchen dieser vier Arten unterschieden werden.

Fig. 1-5 (links): Genital der Männchen verschiedener Beris-Arten (links: Synsternit mit Gonostyli; mittig: Aedeaguskomplex lateral; rechts: Spitze des Aedeaguskomplexes). - 1: Beris chalybata (FORSTER, 1771) [17.VIIl.1997, D, Niedersachsen, Ostfriesland, Ihlower Forst]; - 2: Beris geniculata CurTIS, 1830 [18. V.2002, D, Niedersachsen, Harz, Andreasbergertal]; - 3: Beris hauseri spec. nov. [Paratypus, 23.V.1992, D, Bayern, Alter Einfang, Kellerbach]; - 4: Beris latifacies Nagatomi \& TanakA, 1972 [Paratypus, 04.VII.1963, Japan, Kai, Senjodake]; - 5: Beris strobli Dušek \& Rozkošný, 1968 [18.V.2002, D, Niedersachsen, Harz, Andreasbergertal]. 
Einfach ist die Bestimmung anhand der Genitalabbildungen 1-5 möglich. Die Weibchen von Beris hauseri können in Mitteleuropa aufgrund des dunklen Hinterleibs und der breiten Stirn nur mit Beris chalybata und Beris strobli verwechselt werden. Die Unterscheidung der Weibchen dieser drei Arten ist schwieriger und ohne eine Präparation der Genitalfurka vielleicht nicht immer möglich: Beris hauseri hat (meist undeutlich) verdunkelte Femur, am besten ist dies an den apikalen Hinterfemur zu erkennen. Das erste Glied der Vordertarsen ist apikal hellgelb wie die angrenzende Tibia und am Ende schmal dunkelbraun wie die folgenden Tarsenglieder. Beris chalybata hat ganz gelbe Femur und Tibia und außerdem Vordertarsen, die auch basal mit den angrenzenden Vordertibien kontrastieren. Beris strobli hat deutlicher verdunkelte Beine, besonders unterscheiden die braunen Hintertibien und die einheitlich braunen Hintertarsen die Art von Beris chalybata und Beris hauseri. Das Weibchen von Beris hauseri unterscheidet sich in der Genitalfurka durch den geraden basalen Innenrand der Öffnung (Fig. 7) von Beris chalybata und Beris strobli, bei denen der Innenrand deutlich gebogen ist (Fig. 6, 9).

Die nach Material aus Japan beschriebene Beris latifacies Nagatomi \& TANAKA, 1972 unterscheidet sich von Beris hauseri nur durch Merkmale des männlichen Genitals (Fig. 4): Bei Beris latifacies (1) laufen die Paramere stärker auseinander und apikal nicht wieder zusammen, (2) ist der Mittelfortsatz des Syntergits T 7-10 verglichen mit den Spitzen der Gonostyli geringfügig kürzer und (3) sind die Gonostyli stärker und an der Außenseite unregelmäßiger nach innen gekrümmt. An dem einzigen mir vorliegenden Weibchen von Beris latifacies konnte ich keine Merkmale finden, die eine überzeugende Trennung von Beris hauseri ermöglichen, eventuell sind bei einer Untersuchung einer größeren Serie von Tieren entsprechende Merkmale zu finden - am ehesten an der Genitalfurka (Fig. 8).

Bestimmungsschlüissel: Trennung der Männchen der mitteleuropäischen Arten der Gattung Beris Latreille, 1802 mit dunklem Abdomen, deutlich verdunkelten Flügeln, nicht stark verbreiterten Fühlern und überwiegend schwarzer Mesoscutumbehaarung.

(1) Femur und Tibia einheitlich hell orangebraun bis mittelbraun, mit den dunkleren apikalen Tarsengliedern deutlich kontrastierend; Genital entsprechend Fig. 1: Mittelfortsatz des Syntergits T 7-10 U-förmig ausgeschnitten und nicht bis zu den Spitzen der Gonostyli reichend; Paramere apikal zugespitzt und auseinanderlaufend.

B. chalybata (Forster, 1771)

Femur und Tibia dunkelbraun bis schwarz, oft die Knie und der basale Teil der Tibien hellgelb abgesetzt, die dunkel gefärbten Teile entsprechend farblich den gleich gefärbten apikalen Tarsengliedern. 2

(2) Thorax hell blaugrün metallisch glänzend; Augenbehaarung deutlich länger als Scapus; Genital entsprechend Fig. 2: Mittelfortsatz des Syntergits T 7-10 nicht ausgeschnitten und nicht bis zu den Spitzen der Gonostyli reichend; Paramere apikal verbreitert und in kurzer. Spitze endend; Aedeaguskomplex in Lateralansicht kürzer und weniger stark gebogen. ...

B. geniculata CurTis, 1830

Thorax schwarz metallisch glänzend, höchstens mit undeutlichem Grünglanz; Augenbehaarung höchstens so lang wie Scapus. 
(3) Tibia 1 basal zu mindestens einem Viertel hellgelb oder hellbraun, mit apikalem Bereich deutlich kontrastierend; Basitarsus 1 basal mehr oder weniger stark aufgehellt; Flügel braun gefärbt; Genital ensprechend Fig. 3: Mittelfortsatz des Syntergits T 7-10 V-förmig ausgeschnitten und bis zu den Spitzen der Gonostyli reichend; Paramere apikal zugespitzt und nachdem sie vorher auseinanderlaufen wieder zusammenlaufend; Genital insgesamt deutlich breiter als lang. B. hauseri spec. nov.

Tibia 1 in der Regel einheitlich dunkelbraun bis schwarz, höchstens die Knie heller gelb; Basitarsus 1 in der Regel einheitlich schwarz; Flügel dunkebraun gefärbr; Genital entsprechend Fig. 5: Mittelfortsatz des Syntergits T 7-10 U-förmig ausgeschnitten und nicht bis zu den Spitzen der Gonostyli reichend; Paramere apikal zugespitzt und auseinanderlaufend; Genital insgesamt deutlich länger als breit. B. strobli DušEK \& RozKošNú, 1968

Key: To the males of the Middle European species of the genus Beris LATreille, 1802 with dark abdomen, infuscated blackish wings, not thickened antenna and mainly black abdominal pubescence.

(1) femur and tibia uniform light orange-brown to brown, clearly contrasting with the dark blackish tarsi; genitalia as figure 1: median process of syntergite T 7-10 U-shaped and not reaching the tip of gonostyli; parameres pointed and divergent apically.

B. chalybata (Forster, 1771)

femur and tibia dark brown to black, tibia as dark as the apical tarsomeres, cuticle at junction of femur and tibia often light yellow..... 2

(2) thorax shining metallic light blue-greenish; hairs on the eye clearly longer than scapus; genitalia as figure 2 : median process of syntergite T 7-10 without incision and not reaching the tip of the gonostylus; paramere broadened apical and ending in a short pointed tip; aedeagal complex in lateral view shorter and less curved. B. geniculata CurTis, 1830 thorax shining metallic black, at most slightly shining metallic green; hairs on eye shorter or as long as the scapus.

3

(3) tibia 1 at least one quarter light yellow or light brown at base, distinctly contrasting with the apical part of the tibia; basitarsus 1 basally more or less lightened; wings brown dusted; genitalia as figure 3: median process of syntergite $T$ 7-10 V-shaped and reaching the tip of the gonostylus; paramere pointed, divergent basally and converging apically; genitalia broader than long...... $B$. hauseri spec. nov.

tibia 1 completely dark brown or black, sometimes with lighter cuticle near junction of femur and tibia; basitarsus 1 usually uniform dark brown; wings blackish brown dusted; genitalia as figure 5: median process of syntergites T 7-10 U-shaped and not reaching the tip of the gonostylus; paramere pointed and divergent only; genitalia longer than broad.

B. strobli DušeK \& RozKošný, 1968

\section{Diskussion}

Aus der Sammlung des Naturhistorischen Museum der Benediktiner-Abtei des Klosters Admont (NMBA) liegt mir der Holotypus von Beris strobli vor: 1 i mit folgenden Etiketten: (1) "Beris / obscura Strobl / Dušek et / Rozkošný det." ; (2) "Typen - Exemplar $/$ rev. G. Morge 19" [rotes Etikett]; daneben auf gesonderter Nadel: (3) "Beris strobli / Dušck et Rozkošný / new name, 1968: 295 / [obscura Strobl, praeocc.] / Det. / N. E. Woodley 1980". Dem Exemplar fehlen beide Fühler, der linke Flügel, beide Hinterbeine und das Abdomen. Aufgrund der breiten Stirn, der deutlich verdunkelten Femora und 
Tibia und der einheitlich dunklen Vordertarsen ist die Zuordnung der Art zu dem hier vertretenen Konzept von Beris strobli möglich.

Ein Männchen von Beris strobli aus der Sammlung des Staatlichen Naturkundemuseum Stuttgarts (SMNS) trägt folgende Etiketten: (1) "Präsuraalp En- / gadin 16.VI.1966 / Lindner leg."; (2) "Beris chalybeata / engadinica Lind / Lindner det."; (3) "Typus / Lindner / 1967" [rote Schrift]. Hierbei handelt es sich um einen unpublizierten Manuskriptnamen.

RozkošnÝ \& Nartshun (1988) führen Beris latifacies Nagatomi \& TANAKa, 1972 als Synonym von Beris strobli; Woodley (2001) übernimmt diese Beurteilung. Mir liegen drei Paratypen $\left(20^{*}, 1\right.$ o $)$ der Art vor, die zusammen mit dem Holotypus gesammelt wurden (coll. British Museum for Natural History). Nach den zuvor genannten Merkmalen unterscheidet sich Beris latifacies von Beris hauseri und Beris strobli nach Merkmalen des männlichen Genitals und muss als valide Art geführt werden (status rev.).

Ein Vergleich der Verbreitung von Beris hauseri und Beris strobli ist heute kaum möglich, da das faunistischen Meldungen zugrunde liegende Material dazu revidiert werden muss. Mir liegen folgende Beleg von Beris strabli vor:

Deutschland: $2 \sigma^{\circ} \sigma^{\circ}$ (Bayern, Regierungsbezirk Kalkenried, NSG "Am unteren Stein", leg. Blank, coll Hauser); $1 \mathrm{mmm}$ (27.V.-01.VI.1948, Bayern, Ammergauer Berge, Frieder-Gebiet, 1700-2000 m, leg. F. Daniel \& J. Wolfsberger, coll. ZSMC); $60^{0}$ व, 11 우 우 (18.V.2002, Niedersachsen, Harz, Andreasbergertal/Leimenztal NW Zorge, leg. et coll. Stuke); $50^{*} \sigma^{*}, 11$ 우 (dito); 1 ㅇ (dito, aber leg. et coll. van Steenis); 19 (19. V.2002, Niedersachsen, Hirseteich, $1 \mathrm{~km} \mathrm{NW}$ Walkenried, leg. et coll. van Steenis); 9 우 우 (18.V.2002, Niedersachsen, Harz, „Neuer Teich“ bei Zorge, leg. et coll. Stuke); 1 ㅇ (07.V.2000, Niedersachsen, Kaiserweg, ab B24 bis Teiche, SW Braunlage leg. et coll. Stuke); 1 क (15.V.-01.VI.2002, Sachsen, Weißeritzkreis, NSG „WeißeritzwiesenSchellerhau", 712 m NN, leg. et. coll. Kehlmaier); Österreich: 1 \& (28.V.1995, Lunz/S., Kotbergtal, leg. Ressl, coll. Hauser); Schweiz: 1 ㅇ (16.VI.1966, Sils, Fextal, leg. Lindner, coll. SMNS); $10^{*}$ (24.VI.1967, dito); $20^{*}$ (16.VI.1966, Präsuraalp, Engadin, leg. Lindner, coll. SMNS); $20^{\circ}$ (16.VI.1960, Engadin, Val Fex, leg. Lindner, coll, SMNS); Tschechien: $10^{\star}$ (25.VI.1992, Jesseníky, Karlov p. P. marshes, leg. et coll. Rozkošný); 1 q (09.VI.1975, Hrubý Jeseník, Domášov-Bêlá p. Pr., leg. et coll. Rozkošný); $3 \sigma^{\top} \sigma^{\star}$ (31. V.1984, Krkonoše Mts., Snêžkou, Obrí Dul., 900 m NN, leg. et coll. Rozkošný); 1 \& (31.V.1984, Krkonoše Mts., Jánské Láznê, $600 \mathrm{~m} \mathrm{NN}$, leg. et coll. Rozkošný); Ungarn: 10 (02.V.1993, Bakony Gebirge, Fenyölö, Umgebung Ziro, leg. et coll. Hauser).

Diese Nachweise deuten auf eine weite Verbreitung in Mitteleuropa hin. Zumindest in Mitteleuropa ist Beris strobli eine Art der Mittelgebirge, während Beris hauseri auch im Flachland verbreitet sein kann. Bei der unter "Beris strobli" geführten nearktischen Art wird es sich nach den Genitalabbildungen von WoodeEy (1981) um Beris hauseri handeln, die damit als holarktische Art interpretiert werden muss. Beris latifacies ist bislang nur in der Typenserie aus Japan bekannt geworden.

Aufgrund eigener Beobachtungen der Imagines von Beris strobli halte ich es für möglich, dass sich die Larven in Petasites hybridus entwickeln, wie es schon von einer Reihe anderer Beris-Arten bekannt ist (RozKošnÝ 1982, STUKE 2003). Zur Larvalbiologie von Beris hauseri und Beris latifacies liegen keine Hinweise vor. 
Schluss

Die einleitend genannten Probleme mit der Bestimmung der tatsächlich zu Beris strobli gehörenden Tiere aus dem Harz sind nun zu klären: Dušsk \& RozkošNý (1968) und später RozkošnÝ (1982) trennen die beiden Arten Beris strobli und Beris hauseri nicht.

Bei der Beschreibung der Beinfärbung gehen sie von einer variablen Art aus: "Die Art ist hinsichtlich der gelb gefärbten Beinteile ziemlich variabel". Die Weibchen von Beris hauseri werden nach der Tabelle von DušEK \& RozKоšNÝ (l. c.) Beris chalybata zugeordnet.

Die Genitalabbildungen in den Arbeiten von Dušek \& RozkošnÝ (l. c.) und RozkošNÝ (1. c.) zeigen aber das männliche Genital von Beris hauseri. Mir liegt das Material, das der Originalbeschreibung zugrunde liegt, zum Teil vor. Darunter befinden sich auch die Tiere, nach denen die Genitalabbildungen angefertigt wurden und diese gehören zu Beris hauseri. In den Niederlanden ist bisher nur Beris hauseri nachgewiesen und unter dem Namen Beris strobli geführt worden. Mit dem Bestimmungsschlüssel von BrUGGe (2002) ist eine Bestimmung der echten Beris strobli daher nicht möglich.

\section{Danksagung}

Dr. Jürgen Götze (Deutschland, Berlin) ermöglichte das Entleihen des Holotypus von Beris strobli aus der Sammlung Strobl (Österreich, Naturhistorischen Museum der Benediktiner-Abtei des Klosters Admont). Dr. Hiroshi Shima (Japan, Fukuoka) und Prof. Dr. Akira Nagatomi (Japan, Kagoshima) machten mir Paratypen von Beris latifacies zugänglich. Christian Kehlmaier (Deutschland, Dresden) und Wouter van Steenis (Niederlande, Utrecht) stellten Material aus ihren Privatsammlungen zur Verfügung; Wolfgang Schacht (Bayern, München) entlieh Material aus der Zoologischen Staatssammlung.

Ben Brugge (Amsterdam), Martin Hauser (Urnana), Prof. Dr. Rudolf Rozkošný (Brno) und Dr. Norman E. Woodley (Washington) danke ich für die vielfältigen geduldigen Hilfestellungen, das Entleihen von Material und die vielen wertvollen Diskussionsbeiträge ohne die die vorliegende Arbeit kaum möglich gewesen wäre.

\section{Literatur}

BrugGe, B. 2002: Wapenvliegen Tabel. - Utrecht: Jeudbondsuitgeverij: 94 S.

Dušek, J. \& Rozkošnú, R. 1968: Beris strobli nom. nov. (Diptera, Stratiomyidae). - Reichenbachia 10: 293-298; Dresden.

Kassebeer, C. F. 1996: Eine neue Art der Gattung Beris Latreille, 1802 aus Marokko (Diptera, Stratiomyidae). - Studia dipterologica 3: 155-159; Halle.

Nagatomr, A. \& Tanaka, A. 1972: The Japanese Beris (Diptera, Stratiomyidae). - Memoirs of the Faculty of Agriculture, Kagoshima University 8: 87-113; Kagoshima.

RozKošNÝ, R. 1982: A biosystematic study of European Stratiomyidae (Diptera). Volume 1. Introduction, Beridinae, Sarginae and Stratiomyinae. - Series Entomologica 21: 1-401; The Hague.

Rozkošný, R. \& Nartshuk, E. P. 1980: Two new species of Beris, with a key to the Palearctic species of the genus (Diptera, Stratiomyidae). - Acta entomologica bohemoslovaca 77: 408-418; Prag.

Rozkošnú, R. \& Nartshuk, E. P. 1988: Stratiomyidae. - In: Sóos, A. \& PApp, L. (eds.): Catalogue of Palearctic Diptera. Volume 5. - Amsterdam-Oxford-New York-Tokyo: Elsevier, $446 \mathrm{~S}$.

STUKE, J.-H. 2003: Die Stratiomyidae und Xylomyidae (Diptera) Niedersachsens und Bremens. - Braunschweiger Naturkundliche Schriften 6: 831-856; Braunschweig. 
Woodley, N. E. 1981: A revision of the Nearctic Beridinae (Diptera: Stratiomyidae). - Bulletin of the Museum of Comperative Zoology 149: 319-367; Cambridge.

Woodley, N. E. 2001: A world catalogue of the Stratiomyidae (Insecta: Diptera). - Myia. The International Journal of the North American Dipterists' Society 11: 1- 475; Washington.

YANG, D. \& NAGATOMI, A. 1992: A study on the Chinese Beridinae. - South pacific Study 12: 129-178; Kagoshima.

\section{Anschrift des Verfassers:}

Dr. Jens-Hermann Stuke

Brunnenstraße 28

D-26789 Leer

jstuke@zfn.uni-bremen.de

\section{Buchbesprechung}

Andersen, N. M. \& WeIR, T. A.: Australian Water Bugs. Their Biology and Identification (Hemiptera - Heteroptera, Gerromorpha \& Nepomorpha). - Denmark: Apollo Books \& Australia: Csiro Publishing, 2004. - 344 S., zahlr. Fig. (Entomonograph; 14) ISBN 0643090517.

Die zu den Insekten mit unvollständiger Verwandlung gehörenden Wasserwanzen (Ordnung Hemiptera) sind weltweit verbreitet. Man unterscheidet Gerromorpha, halbaquatische Wanzen, die auf der Wasseroberfläche leben und Nepomorpha, echte Wasserwanzen, die unter Wasser leben. Sie besiedeln nahezu alle aquatischen Habitate: kleine, nur temporär wasserführende Tümpel, Teiche und Seen, Bäche und Flüsse, Süßwasser sowie Mangrovengewässer, Lachen der Gezeitenzonen und sogar den offenen Ozean. Einige Farbtafel im Buch von Nils M. Andersen und Tom A. Weir illustrieren diese unterschiedlichen Lebensräume.

Wasserwanzen sind hauptsächlich Räuber und Aasfresser, die von allem leben, was sie überwältigen können: Kleinkrebse, Insekten, Kaulquappen und kleine Fische. Sie sind wichtige Komponenten in aquatischen Ökosystemen und können als Indikator der biologischen Qualität von Gewässern dienen. Einige Arten, z.B. solche, die Moskitolarven jagen und andere Arten, die als Fischnahrung dienen, haben auch ökonomische Bedeutung. Aufgrund ihrer unterschiedlichen Biologie und der Möglichkeit die Tiere relativ einfach in ihren Biotopen beobachten zu können, sind sie exzellente Modellorganismen für die Evolutionsbiologie, die Ökologie und den Naturschutz.

Das vorliegend Handbuch gibt einen Überblick über alle 15 Familien, 17 Unterfamilien und 55 Gattungen, die von Australien, Tasmanien und den angrenzenden Inseln bekannt sind. Ausführungen zur Biologie und Ökologie der Wasserwanzen, ihre Systematik und Phylogenie sind ebenso vorhanden, wie Angaben zum Sammeln, Präparieren und Bestimmen der 261Arten. Ein Bestimmungsschlüssel anhand von charakteristischen Habitaten und auffälligen Verhaltensmustern ermöglichen eine schnelle Annäherung an einige Gruppen.

Die eigentlichen Bestimmungsschlüssel auf über 260 Seiten sind reich illustriert. Detaillierte, vergleichende Zeichnungen erleichtern die Determination und halten die Merkmalsbeschreibungen kurz. Zusätzliche Aufnahmen vom Raster-Elektronen-Mikroskop zeigen teils Habitusbilder, teils Feinstrukturen. Anhand von Verbreitungskarten kann die Bestimmung schließlich verifiziert werden.

Da viele Familien der Wasserwanzen, z. B. Gerridae, Nepidae oder Notonectidae weltweit verbreitet sind, ist das Handbuch nicht nur dem Spezialisten der Gruppe und dem Australien-Reisenden, sondern auch dem europäischen Heteropterologen empfohlen. 